\title{
The withdrawal of the Liverpool Care Pathway in the United Kingdom: what are the implications for Australia?
}

\section{A lack of evidence means that the benefits and harms of end-of-life care pathways remain poorly understood}

ntegrated care pathways are documents that outline the essential steps of multidisciplinary care in dealing with a specific clinical problem. ${ }^{1}$ They can be used to introduce best clinical practice, to ensure that the most appropriate management occurs at the most appropriate time, and that it is provided by the most appropriate health professional. By providing clear instructions, decision support and a framework for clinician-patient interactions, care pathways guide the systematic provision of best evidence-based care. The Liverpool Care Pathway for the Dying Patient (LCP) is an example of an integrated care pathway - it was designed in the United Kingdom in the 1990s to guide care for people with cancer who were in their last days of life and expected to die in hospital. 2,3 This pathway evolved out of a recognised local need to better support nonspecialist palliative care providers to care for patients dying of cancer in their inpatient units. Historically, despite the many people in acute care settings whose treatment intent was palliative, ${ }^{4}$ dying patients in these settings tended to receive insufficient attention from senior medical and nursing staff. ${ }^{5}$ As the quality of end-of-life care was considered inadequate, much could be learned from the way patients were cared for by palliative care services. ${ }^{5}$ The LCP was a strategy to improve this end-of-life care and was based on the care received by those dying in the palliative care setting., ${ }^{2,3}$

The LCP defines 18 goals of care for patients in the last 2 days of life. A range of care standards relating to comfort, psychological and insight concerns, religious and spiritual support, communication with family and others, communication with the primary health care team, and care after death are detailed in the LCP. 2,3 The LCP outlines clear procedures and guidance for the multidisciplinary team to approach care. Over the past decade, the rapid diffusion of the LCP, from being a local solution to being implemented widely, has been phenomenal. This expansion has occurred in the absence of robust evaluation. In the UK, the LCP was actively promoted by the National Health Service and was adopted as standard practice by more than 1800 health care institutions, ${ }^{6}$ with evidence of its use extending to other parts of the world. ${ }^{7}$ Across Australia, several endof-life care pathways (EOLCPs) have been adapted from the LCP. Although there are no existing data indicating a precise number of institutions using them, it is clear that there has been widespread uptake of EOLCPs in Australian acute care hospitals and residential aged care facilities (RACFs). ${ }^{8-15}$ Perhaps based on this widespread adoption, the National Palliative Care Strategy $2010^{9}$ identified the need to support a national roll-out of an integrated EOLCP across all care sectors (primary, acute and aged care).

In 2013, in response to substantial concerns raised by the public and by health professionals, the UK government commissioned an independent review of the LCP, led by Baroness Julia Neuberger. ${ }^{16}$ The panel reviewed multiple sources, including written submissions from the public and health professionals with experience of the LCP, the academic literature and hospital complaints. Based on the evidence provided, the panel determined that the implementation of the LCP was often associated with poor care. ${ }^{16}$ Its report highlighted the complexity around the use of the pathway, specifically highlighting ethical, safety, clinical practice and legal problems, and how poorly dying is diagnosed in clinical care. ${ }^{16}$ Although the intention and end-of-life care principles underpinning the LCP are sound, use of the pathway has extended well beyond the evidence base and despite the lack of any rigorous, prospective evaluation at the time of its widespread uptake. ${ }^{6,7}$ Further, it was emphasised in the report that the LCP cannot and should not replace good clinical practice encompassing empathy, humanity and communication. As a result of the review, the UK government decided to phase out the LCP nationally over the following 6-12 months. ${ }^{17}$

doi: 10.5694/mjal3.10998

A recent update of our Cochrane systematic review ${ }^{6}$ identified four Australian studies (one audit ${ }^{12}$ and three pretest-post-test studies ${ }^{13-15}$ ) evaluating EOLCPs, all of which had been adapted from the LCP. The retrospective audit of 160 hospital inpatients reported potential benefits of an EOLCP in documenting clinician assessments of goals of care as designed by the pathway. ${ }^{12}$ A pilot study of 35 general medical patients reported potential benefits in documentation, medication orders and other aspects of care. ${ }^{13}$ Another study demonstrated that, after the implementation of an EOLCP for residents of six RACFs, a significantly lower proportion of those who were commenced on the EOLCP transferred to hospital at the end of life (2/118) compared with those who were not commenced on the EOLCP (29/135). ${ }^{15}$ The following year, another pretest-post-test study (involving 600 RACF residents before implementation of an EOLCP, and 594 after implementation) did not find any statistically significant differences in the number of deaths in RACFs, deaths in hospitals or transfers to hospitals. ${ }^{14}$

Despite the widespread adoption and policy endorsement of EOLCPs, there has been insufficient rigorous evaluation of the net effect in Australia 
or internationally. The benefits and harms of such pathways, whether intended or unintended, remain poorly understood. ${ }^{6,7}$ The only randomised controlled trial to date, conducted with Italian cancer patients, did not find any significant benefits for improving patient outcomes. ${ }^{18}$ The lack of high-quality health services research before widespread implementation of the LCP in the UK and other countries has created a dilemma for Australia: do the adverse findings from the Neuberger review $^{16}$ apply to Australia and, if so, to what extent? If not, how certain are we? What are the implications of these findings for our National Palliative Care Strategy? And how should the Strategy's recommended action to support the national roll-out of an EOLCP be managed? Rigorous studies should be designed to establish whether the outcomes for dying patients who are placed on an EOLCP are different to those for patients receiving usual care and, if so, to answer several key questions:

- Are the right people put on an EOLCP at the right time in their illness trajectory?

- In which settings should an EOLCP be used?

- Who should have the authority to initiate an EOLCP?

- What specific medical history is required before initiating an EOLCP?

- How senior should the clinicians be and how much of the patient's history should they know before initiating an EOLCP?

- Are there any differences in outcomes when comparing different EOLCPs?

There remains an urgent need for large Phase III studies thoroughly measuring these outcomes in Australia, and subsequent monitoring of the outcomes if widespread uptake is supported by well designed, well conducted studies. ${ }^{19}$ The conduct of such studies can be complex and expensive. The National Health and Medical Research Council (NHMRC) and other funding bodies should support research teams that consist of experienced palliative care researchers and clinicians, and an economist. Process and cost-effectiveness outcomes must not be ignored in any future research of EOLCPs. To ensure generalisability, studies should stratify participants according to different care settings, including general acute care, emergency departments, cancer care units, RACFs and specialist palliative care units. Were such studies to produce positive results, careful ongoing evaluation of the outcomes of implementing any revised pathway as it is broadly introduced would be imperative.

While we wait for results from future prospective randomised evaluations, we recommend that the next sensible step for Australia is to contextually review each of the shortfalls or potential adverse effects of the LCP highlighted by the Neuberger review. ${ }^{16}$ Subsequently, actions to avoid these unwanted consequences could be assessed. Importantly, the Neuberger review identified that clinicians need to actively diagnose that a person is dying, and that no obvious reversible or precipitous changes have been overlooked. Once established, the diagnosis needs to be compassionately but clearly communicated to the patient and his or her family. An evidence-based clinical practice guideline on communicating prognosis and end-of-life issues to patients with advanced disease and their caregivers has been published. ${ }^{20}$ It provides health professionals with useful and unambiguous guidance about their role in acknowledging the uncertainty and unreliability of prognostic predictions and the difficulty this creates for patients or caregivers. Failure to communicate clearly was one of the most serious concerns expressed in the Neuberger review.

Australia needs to respond to these concerns that have been so carefully documented at a time when end-of-life awareness continues to rise. There is no room for complacency. Rather than pursuing an endof-life care strategy based on limited evidence, we urge policymakers to continue to invest in building the palliative care capabilities of the Australian health care workforce, extending the reach of specialist palliative care services and building the palliative care evidence base through investing in rigorous research. Ultimately, if the LCP is to be replaced, there needs to be systematic measurement of the benefits and harms generated by such a process. No intervention is without problems, and as a new process is implemented, the unexpected harms it may generate need to be measured so they can be overcome as they arise.

Competing interests: No relevant disclosures.

Provenance: Not commissioned; externally peer reviewed.

1 Rotter T, Kugler J, Koch R, et al. A systematic review and meta-analysis of the effects of clinical pathways on length of stay, hospital costs and patient outcomes. BMC Health Serv Res 2008; 8: 265.

2 Ellershaw J, Foster A, Murphy D, et al. Developing an integrated care pathway for the dying patient. Eur $J$ Palliat Care 1997; 4: 203-207.

3 Ellershaw J, Ward C. Care of the dying patient: the last hours or days of life. BMJ 2003; 326: 30-34.

4 To TH, Greene AG, Agar MR, Currow DC. A point prevalence survey of hospital inpatients to define the proportion with palliation as the primary goal of care and the need for specialist palliative care. Intern Med J 2011; 41: 430-433.

5 Mills M, Davies HT, Macrae WA. Care of dying patients in hospital. BMJ 1994; 309: 583-586.

6 Chan R, Webster J. End-of-life care pathways for improving outcomes in caring for the dying. Cochrane Database Syst Rev 2010; (1): CD008006.

7 Phillips JL, Halcomb EJ, Davidson PM. End-of-life care pathways in acute and hospice care: an integrative review. J Pain Symptom Manage 2011; 41: 940-955.

8 Department of Health (UK). End of life care strategy: promoting high quality care for all adults at the end of life. London: Department of Health, 2008.

9 Australian Government Department of Health and Ageing. National Palliative Care Strategy 2010: supporting Australians to live well at the end of life. Canberra: Department of Health and Ageing, 2010.

10 Department of Health, Government of Western Australia. The WA Icp: a pathway to improved care of the dying in Western Australia. Perth: Department of Health, 2013.

11 Stepanov N, Quinn K, Philip J, Boughey M. End of life care and care pathways in Victoria: report and recommendations. Melbourne: Centre for Palliative Care, 2011.

12 Hardy JR, Haberecht J, Maresco-Pennisi D, Yates P; Australian Best Care of the Dying Network. Audit of the care of the dying in a network of hospitals and institutions in Queensland. Intern Med J 2007; 37: 315-319.

13 Jackson K, Mooney C, Campbell D. The development and implementation of the pathway for improving the care of the dying in general medical wards. Intern Med J 2009; 39: 695-699.

14 Horey DE, Street AF, Sands AF. Acceptability and feasibility of end-of-life care pathways in Australian residential aged care facilities. Med J Aust 2012; 197: 106-109.

15 Reymond L, Israel FJ, Charles MA. A residential aged care end-of-life care pathway (RAC EoLCP) for Australian aged care facilities. Aust Health Rev 2011; 35: 350-356.

16 Neuberger J, Aaronovitch D, Bonser T, et al. More care, less pathway: a review of the Liverpool Care Pathway. London: Department of Health, 2013.

17 Lamb N. The Liverpool Care Pathway should be replaced [media release]. London: Department of Health (UK), 2013. https://www.gov.uk/government/uploads/system/uploads/attachment_data/file/212452/ Press_notice_-_Liverpool_Care_Pathway.pdf (accessed Aug 2013).

18 Craig P, Dieppe P, Macintyre S, et al. Developing and evaluating complex interventions: new guidance. London: Medical Research Council, 2008.

19 Clayton JM, Hancock KM, Butow PN, et al. Clinical practice guidelines for communicating prognosis and end-of-life issues with adults in the advanced stages of a life-limiting illness, and their caregivers. Med $J$ Aust 2007; 186 (12 Suppl): S77-S108. 